\title{
Coupling creep and damage in concrete under high sustained loading: Experimental investigation on bending beams and application of Acoustic Emission technique.
}

\author{
J. Saliba, A. Loukili, and F. Grondin
}

Institut de Recherche en Génie Civil et Mécanique (GeM), UMR-CNRS 6183, Centrale Nantes, BP

92101, 44321 Nantes, France

\section{Abstract}

Creep and damage in concrete govern the long-term deformability of concrete. Thus, it is important to understand the interaction between creep and damage in order to design reliable civil engineering structures subjected to high level loading during a long time. Many investigations have been performed on the influence of concrete mixture, the effect of the bond between the matrix and the aggregates, temperature, aging and the size effect on the cracking mechanism and fracture parameters of concrete. But there is a lack of results on the influence of the creep loading history. In the present paper, an experimental investigation on the fracture properties of concrete beams submitted to three point bending tests with high levels of sustained load that deals with creep is reported. The results aim first to investigate the ranges of variation of the time response due to creep damage coupled effects under constant load and secondly to evaluate the residual capacity after creep.

For this purpose a series of tests were carried out on geometrically similar specimens of size 100x200x800mm with notch to depth ratio of 0.2 in all the test specimens. The exchange of moisture was prevented and beams were subjected to a constant load of $70 \%$ and $90 \%$ of the maximum capacity.

Three point bending test were realized on specimen at the age of 28 days to determine the characteristics of concrete and the maximum load so we could load the specimens in creep. Threepoint bend creep tests were performed on frames placed in a climate controlled chamber [1]. Then after four months of loading, the beams subjected to creep were removed from the creep frames and then immediately subjected to three-point bending test loading up to failure with a constant loading rate as per RILEM-FMC 50 recommendations.

The residual capacity on the notched beams and the evolution of the characteristics of concrete due to the basic creep was considered. The results show that sustained loading had a strengthening effect on concrete, probably because of the consolidation of the hardened cement paste. The influence of creep on fracture energy, fracture toughness, and characteristic length of concrete is also studied. The fracture energy and the characteristic length of concrete increases slightly when creep occurs prior to failure and the size of the fracture process zone increases too. The load-CMOD relationship is linear in the ascending portion and gradually drops off after the peak value in the descending portion. The length of the tail end portion of the softening curve increases with beams subjected to creep. Relatively more ductile fracture behavior was observed with beams subjected to creep.

The contribution of non-destructive and instrumental investigation methods is currently exploited to check and measure the evolution of some negative structural phenomena, such as micro-and 
macro-cracking, finally resulting in a creep-like behaviour. Among these methods, the nondestructive technique based on acoustic Emission proves to be very effective, especially to check and measure micro-cracking that takes place inside a structure under mechanical loading. Thus as a part of the investigation quantitative acoustic emission techniques were applied to investigate microcracking and damage localization in concrete beams. The AE signals were captured with the AE WIN software and further analyzed with Noesis software analysis of acoustic emission data. AE waveforms were generated as elastic waves in concrete due to crack nucleation. And a multichannel data acquisition system was used to record the AE waveforms. During the three point bending tests, quantitative acoustic emission (AE) techniques were used to monitor crack growth and to deduce micro fracture mechanics in concrete beams before and after creep. Several specimens are experimented in order to match each cluster with corresponding damage mechanism of the material under loading. At the same time acoustic emission was used to investigate characteristic of the fracture process zone (length width and macro crack propagation) [4] and to measure energy associated with fracture of concrete test specimens [5]. It is further suggested that AE energy release can be related to actual crack formation energy and the study of the nature of waves release may explain different mechanism as friction and other internal energy dissipation or toughening mechanisms.

In addition more experiments were conducted using acoustic emission with the three point bending test and $\mathrm{AE}$ with specimen under creep so that the analysis could be capable of detecting events during creep. Then acoustic emission is analyzed and illustrated the correlation between basic displacement and total number of acoustic events or in other terms the damage occurring inside the material under creep $[2,3]$.

\section{Keywords:}

concrete beams, creep, damage, acoustic techniques.

1 M.Omar, A.Loukili, G.Pijaudier-cabot, Y.Le Pape, Creep-damage coupled effects:experimental investigation on bending beams with various sizes, Journal of materials in civil engineering, 21, (2009)

2 E.Verstrynge, L.Schueremans, D.V.Gement, M.Wevers, Monitoring and predicting masonry's creep failure with the acoustic emission technique, NDT and E International, (2009)

3 P.Rossi, N.Godart, J.L.Robert, J.P.Gervais, D.Bruhat, Investigation of the basic creep of concrete by acoustic emission, Materials and Structures, 27, (1994)

4 H.Hadjab, J.Fr.Thimus, M.Chabaat, The use of acoustic emission to investigate fracture process zone in notched concrete beams, Current science, 93 (2007)

5 E.N.Landis, L.Baillon, Experiments to relate acoustic emission energy to fracture energy of concrete, Journal of engineering mechanics, (2002) 